\title{
Heterochromatin diversity and its co-localization with $5 S$ and 45S rDNA sites in chromosomes of four Maxillaria species (Orchidaceae)
}

\author{
Juliano S. Cabral ${ }^{1}$, Leonardo P. Felix ${ }^{2}$ and Marcelo Guerra ${ }^{3}$ \\ ${ }^{1}$ Departamento de Biologia, Universidade Federal Rural de Pernambuco, Recife, PE, Brazil. \\ ${ }^{2}$ Departamento de Fitotecnia, Universidade Federal da Paraíba, Areia, PB, Brazil. \\ ${ }^{3}$ Departamento de Botânica, Universidade Federal de Pernambuco, Recife, PE, Brazil.
}

\begin{abstract}
We investigated four orchids of the genus Maxillaria (M. discolor, M. acicularis, M. notylioglossa and M. desvauxiana) in regard to the position of heterochromatin blocks as revealed using chromomycin $\mathrm{A}_{3}(\mathrm{CMA})$ and 4'-6-diamidino-2-phenylindole (DAPI) fluorochrome staining and 5S and 45S rDNA sites using fluorescence in situ hybridization (FISH). The species showed differences in chromosome number and a diversified pattern of $\mathrm{CMA}^{+}$and $\mathrm{DAPI}{ }^{+}$bands, including heteromorphism for $\mathrm{CMA}^{+}$bands. The $5 \mathrm{~S}$ and $45 \mathrm{~S}$ rDNA sites also varied in number and most of them were co-localized with $\mathrm{CMA}^{+}$bands. The relationship between $5 \mathrm{~S}$ rDNA sites and $\mathrm{CMA}^{+}$bands was more evident in $M$. notylioglossa, in which the brighter $\mathrm{CMA}^{+}$bands were associated with large $5 \mathrm{~S}$ rDNA sites. However, not all $5 \mathrm{~S}$ and $45 \mathrm{~S}$ rDNA sites were co-localized with $\mathrm{CMA}^{+}$bands, probably due to technical constraints. We compare these results to banding data from other species and suggest that not all blocks of tandemly repetitive sequences, such as $5 \mathrm{~S}$ rDNA sites, can be observed as heterochromatin blocks.
\end{abstract}

Key words: heterochromatin, CMA and DAPI, 45S and 5S rDNA, Maxillaria.

Received: September 14, 2005; Accepted: February 14, 2006;

\section{Introduction}

Secondary constrictions and heterochromatic regions are the most intensively studied and best known chromosome markers in both plants and animals. Secondary constrictions can be observed by conventional chromosome staining techniques, but are more easily identified by silver nitrate (Ag) staining or in situ hybridization with $45 \mathrm{~S}$ rDNA probes (see e.g. Brasileiro-Vidal et al., 2003). These constrictions are largely known as $45 \mathrm{~S}$ rDNA location sites, although smaller or less active 45S rDNA sites may exist in chromosomes which apparently do not form secondary constrictions (Guerra et al., 1996). On the other hand, heterochromatic regions are generally identified by either Cbanding techniques or direct staining with base-specific fluorochromes, such as 4-6-diamidino-2-phenylindole (DAPI), Hoechst 33258, chromomycin A3 (CMA), mythramycin, quinacrin, and others (Schmid and Guttenbach, 1988; Sumner, 1990; Guerra, 2000). The reaction of these fluorochromes with the chromosomes depends mainly on the nitrogen base composition of the DNA molecule, in such a way that each region of the chromosome may show a positive $(+)$,

Send correspondence to Marcelo Guerra. Rua Nelson Chaves S/N, Cidade Universitária, 50670-420 Recife, Pernambuco, Brazil. E-mail: msfguerra@hotmail.com. negative (-) or neutral (0) reaction with a given fluorochrome (Schweizer, 1981). In plants, the most used fluorochromes are CMA, which preferentially stains GC-rich DNA, and DAPI, which preferentially stains AT-rich regions. Using these two fluorochromes, heterochromatin blocks can be characterized as $\mathrm{CMA}^{+} / \mathrm{DAPI}^{-}$(GC-rich), $\mathrm{CMA}^{-} / \mathrm{DAPI}^{+}$ (AT-rich) or neutral $\left(\mathrm{CMA}^{0}, \mathrm{DAPI}^{0}\right)$ for either one or both fluorochromes (Guerra, 2000).

The origin of the differential staining of heterochromatin after C-banding is still unclear, although it is certainly related to one of the most universal characteristics of heterochromatin, i.e. the presence of one or more families of tandemly repetitive DNA sequences. Such repeat families can be recognized by different fluorochromes or by fluorescent in situ hybridization (FISH) (Cuadrado and Schwarzacher, 1998; Shibata et al., 2005).

As the tandemly repetitive sequences are generally short and non-coding, heterochromatin is predominantly empty of genes. However, the heterochromatin adjacent to the secondary constrictions is an exception, being constituted of tandemly repetitive $45 \mathrm{~S}$ rRNA genes, which forms large rDNA blocks identified as positive regions for Cbanding and CMA staining (Fuchs et al., 1998; Guerra and Felix, 2000). In a different manner, $5 \mathrm{~S}$ rRNA genes are also tandemly organized, but they do not form constrictions in 
prophase or metaphase chromosomes and rarely are visible as heterochromatin blocks (Fuchs et al., 1998; Martins and Wasko, 2004).

During a cytotaxonomic analysis of about 30 Maxillaria Ruiz \& Pavón species (unpublished data), four species belonging to different sections of the genus were selected to investigate the diversity and distribution of CMA or DAPI heterochromatin blocks and the position of 5S and 45S rDNA sites. Maxillaria is the fifth largest American orchid genus, harboring about 420 tropical species (Pabst and Dungs, 1975). Despite such high diversity in the neotropical flora, the cytology of the genus is almost unknown in that chromosome number data is available for only six species (Felix and Guerra, 2000). The four species analyzed in our study showed differences in chromosome number and revealed a diversified pattern of $\mathrm{CMA}^{+}$and $\mathrm{DAPI}^{+}$bands, having $5 \mathrm{~S}$ and $45 \mathrm{~S}$ rDNA sites co-localized with $\mathrm{CMA}^{+}$bands. The meaning of these results is discussed regarding the cytological characterization of heterochromatin.

\section{Materials and Methods}

We collected four Maxillaria species in field excursions in different Brazilian states, the species analyzed being: M. acicularis Herb. (voucher 10830 EAN), collected in Ouro Preto, Minas Gerais state); M. notylioglossa Rchb. f. (voucher 10824 EAN), collected in Domingos Martins, Espírito Santo state; M. desvauxiana Rchb.f. (voucher 10914 EAN) and M. discolor (Lodd. ex Lindl.) Rchb. f. (voucher 10913 EAN), both collected in Jaqueira, Pernambuco state. The species were maintained in cultivation at the Experimental Garden of the Department of Botany of the Federal University of Pernambuco. Vouchers of all species are deposited in the EAN herbarium, Federal University of Paraíba.

For cytological analyses, root tips were pre-treated with $0.002 \mathrm{M}$ 8-hydroxyquinoline at $8^{\circ} \mathrm{C}$ for $20 \mathrm{~h}$, fixed in ethanol/glacial acetic acid 3:1 (v/v) for two hours at room temperature and stored at $-20{ }^{\circ} \mathrm{C}$. Root tips were digested using a solution containing $2 \%$ cellulase and $20 \%$ pectinase (both w/v) for $90 \mathrm{~min}$ at $37{ }^{\circ} \mathrm{C}$ and the meristem dissected in $45 \%(\mathrm{v} / \mathrm{v})$ aqueous acetic acid, squashed under a coverslip (subsequently removed by freezing in liquid nitrogen), air-dried and then aged for three days.

The aged preparations were stained at room temperature with $0.5 \mathrm{CMA} \mathrm{mg} \mathrm{mL}^{-1}$ for $60 \mathrm{~min}$ and then $2 \mu \mathrm{g} \mathrm{mL}^{-1}$ DAPI for 30 min before being mounted in $1: 1(\mathrm{v} / \mathrm{v})$ Mcllvaine's pH 7.0 buffer/glycerol containing $2.5 \mathrm{mM}$ $\mathrm{MgCl}_{2}$ and aged for at least three days for fluorochrome stabilization. The best slides were analyzed using a Leica DMLB microscope and cell images captured with a Cohu digital camera using the QFISH software (Leica). Afterwards, the slides were de-stained at room temperature in 3:1 (v/v) ethanol:acetic acid for $30 \mathrm{~min}$, dehydrated in abso- lute ethanol for two hours at room temperature, air-dried and stored at $-20{ }^{\circ} \mathrm{C}$ until being subjected to fluorescence in situ hybridization (FISH) experiments.

For 45S rDNA FISH analyses we used the Arabidopsis thaliana $\mathrm{R} 2$ probe (Wanzenböck et al., 1997) which was labeled by nick translation with digoxigenin, while for locating 5S rDNA we used the Lotus japonicus D2 probe (Pedrosa et al., 2002) which was labeled by nick translation with biotin. The hybridization mixture contained $50 \%(\mathrm{v} / \mathrm{v})$ formamide, $10 \%(\mathrm{v} / \mathrm{v})$ dextran sulfate, $2 \mathrm{x}$ SSC and 2 to $5 \mathrm{ng} \mu \mathrm{L}^{-1}$ of each probe. The preparations were hybridized in situ overnight and the stringent wash was performed in $0.1 \times \mathrm{SSC}$ at $42{ }^{\circ} \mathrm{C}$. The final stringency was $76 \%$. The $45 \mathrm{~S}$ rDNA sites were detected with sheep anti-digoxigenin-FITC (avidin-fluorescein isothiocyanate) (Roche 1207741) plus anti-sheep-FITC (Dako F0135). The 5S rDNA sites were detected with mouse anti-biotin (Dako M0743) in combination with anti-mouse-TRITC (tetramethyl rhodamine isothiocyanate) (Dako R0270). The chromosomes were counterstained with $2 \mu \mathrm{g} \mathrm{mL}^{-1}$ DAPI, mounted in Vectashield (Vector) and analyzed using the Leica microscope as described above.

\section{Results}

All the species investigated exhibited stable chromosome numbers and had similar-sized chromosomes, about $4.5 \mu \mathrm{m}$ long. After CMA/DAPI double-staining, some chromosome regions were $\mathrm{CMA}^{+} / \mathrm{DAPI}^{-}$(i.e. brighter with CMA and duller with DAPI), whereas others were $\mathrm{CMA}^{-}$/ $\mathrm{DAPI}^{+}$or neutral with one of the fluorochromes $\left(\mathrm{CMA}^{0}\right.$ or $\left.\mathrm{DAPI}^{0}\right)$. We observed chromosome pairs with both $\mathrm{CMA}^{+}$ and DAPI $^{+}$bands only in $M$. acicularis and $M$. notylioglossa. Heteromorphism for $\mathrm{CMA}^{+}$bands was detected in all species except in $M$. discolor. The largest blocks of $5 \mathrm{~S}$ rDNA always coincided with $\mathrm{CMA}^{+} / \mathrm{DAPI}^{-}$ bands, while the sites of $45 \mathrm{~S}$ rDNA always co-localized with $\mathrm{CMA}^{+}$blocks of different fluorescence intensity (Figure 1). On the other hand, DAPI ${ }^{+}$bands and rDNA sites were never found closely associated. The position of the fluorescent bands and rDNA sites and the number of chromosomes without band differentiation are shown in Figure 2, although chromosome size and centromere position are not indicated because they could not be consistently identified. A brief karyotype description of each species is presented below.

Maxillaria discolor $(2 n=42)$ exhibited four terminal $\mathrm{CMA}^{+} / \mathrm{DAPI}^{-}$bands (arrows in Figure 1a-c), two proximal $\mathrm{CMA}^{+} / \mathrm{DAPI}^{0}$ bands (arrowheads in Figure 1a-c) and at least 16 proximal $\mathrm{CMA}^{-} / \mathrm{DAPI}^{+}$bands (half of them in metacentric chromosomes and half in submetacentric to acrocentric chromosomes - see Figure 2). Four 45S rDNA sites and two 5S rDNA sites were also observed (Figure 1d). After sequential staining the $45 \mathrm{~S}$ site co-localized with 
the terminal $\mathrm{CMA}^{+} / \mathrm{DAPI}^{-}$bands and the $5 \mathrm{~S}$ rDNA sites with the proximal $\mathrm{CMA}^{+} / \mathrm{DAPI}^{0}$ bands.

Maxillaria acicularis $(2 \mathrm{n}=38)$ exhibited 18-20 proximal $\mathrm{CMA}^{-} / \mathrm{DAPI}^{+}$bands, all localized in submetacentric to acrocentric chromosomes, and at least four $\mathrm{CMA}^{+} /$ DAPI bands (Figure 1e). In some cells up to three addi- tional $\mathrm{CMA}^{+} / \mathrm{DAPI}^{-}$bands were also observed (Figure $1 \mathrm{~h}$ ). After FISH, four $45 \mathrm{~S}$ rDNA sites and four $5 \mathrm{~S}$ rDNA sites were visible (Figure 1f, g), among these one locus of each different rDNA family was heteromorphic in size. The strongest signals were produced by three $45 \mathrm{~S}$ rDNA sites and one $5 \mathrm{~S}$ rDNA site which clearly coincided with the four

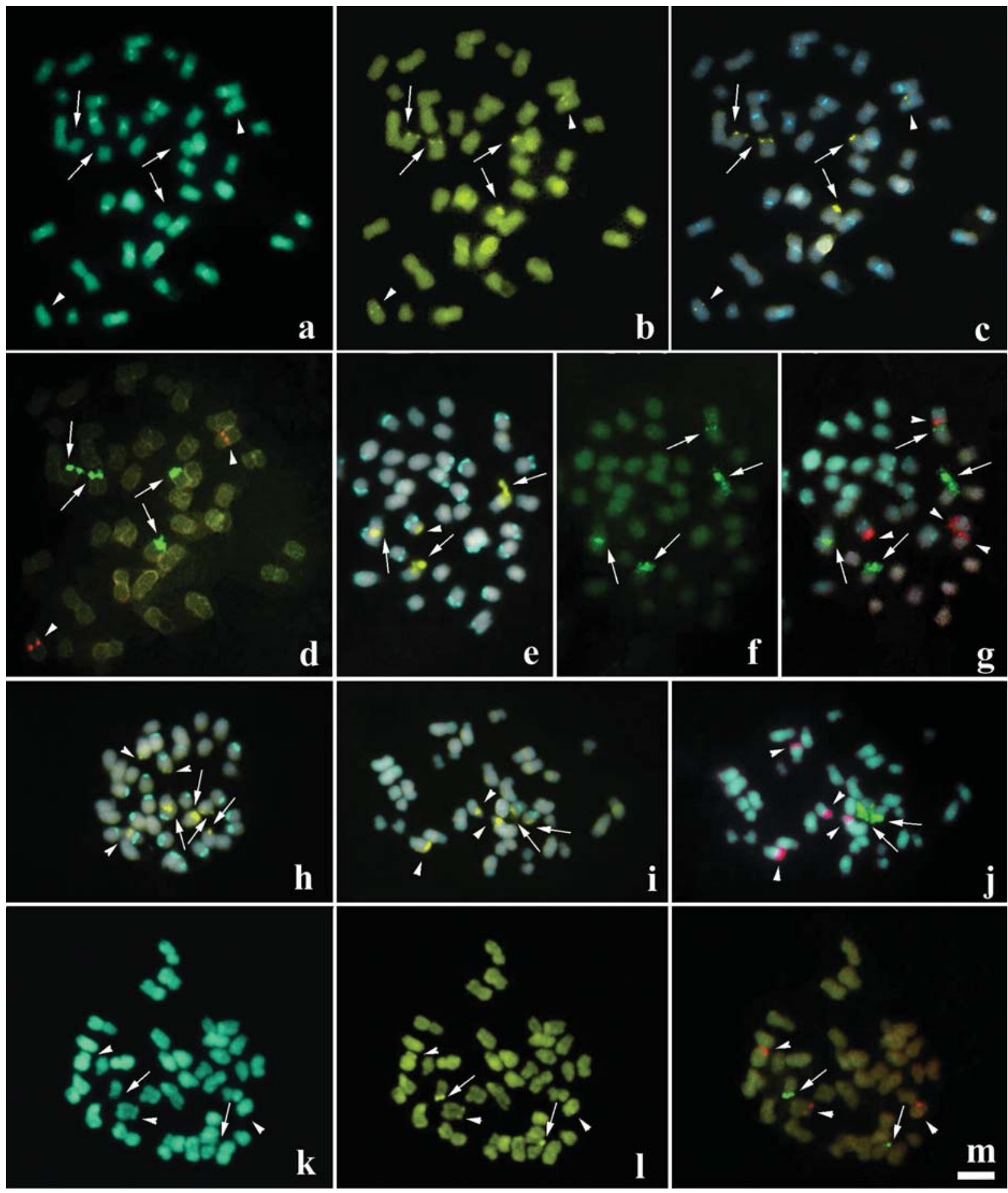

Figure 1 - Metaphase cells of Maxillaria discolor (a-d), M. acicularis (e-h), M. notylioglossa (i,j), and M. desvauxiana (k-m) showing CMA bands (yellow), DAPI ${ }^{+}$bands (blue), 5S and 45S rDNA sites (detected in red with TRITC, and in green with FITC, respectively). a, k, DAPI; b, l, CMA; c, h, i,

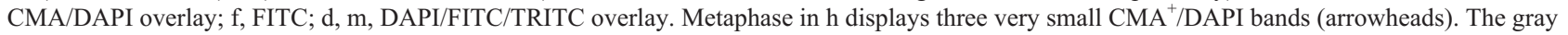
color in some photographs (c, e, g-j) is due to the overlay of different colors. Arrowheads and arrows indicate the sites of 5S and 45S rDNA sites, respectively. Bar in $\mathrm{m}$ corresponds to $5 \mu \mathrm{m}$. 


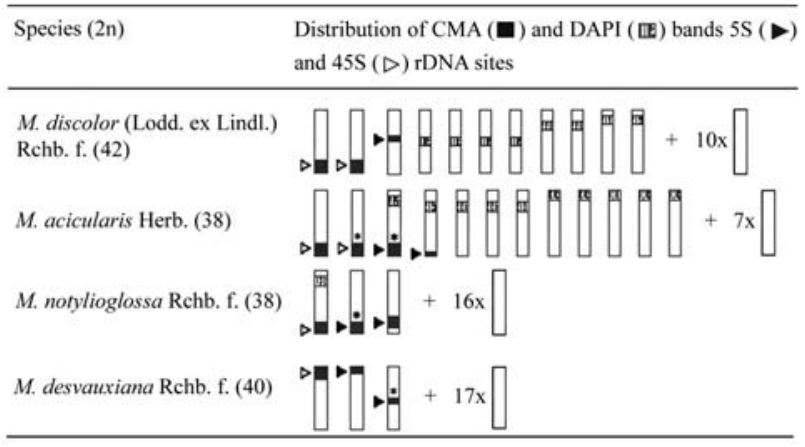

Figure 2 - Chromosomal distribution of heterochromatic bands and $5 \mathrm{~S}$ and $45 \mathrm{~S}$ rDNA sites in the haploid chromosome complement of four Maxillaria species. Centromere position and chromosome size are not indicated, but the chromosomes are represented with the long arms at the bottom. Therefore, all chromosome bands in or close to the top are proximal bands of acrocentric chromosomes. Wherever band-size heteromorphism was observed (indicated by an asterisk) only the largest band variant was represented.

largest $\mathrm{CMA}^{+} / \mathrm{DAPI}^{-}$bands (arrows and arrowhead in Figure 1e). Therefore, these four $\mathrm{CMA}^{+}$bands are distributed in three different chromosome pairs: one pair homozygous for a large 45S rDNA site, one pair heterozygous for a large $45 \mathrm{~S}$ rDNA and a third pair heterozygous for a large $5 \mathrm{~S}$ rDNA site. The three smallest 5S rDNA sites also seemed to co-localize with the smallest $\mathrm{CMA}^{+} / \mathrm{DAPI}^{-}$bands, since both marks were located in a similar position on chromosomes with proximal DAPI bands. The smallest $45 \mathrm{~S}$ rDNA site was probably too small to generate a $\mathrm{CMA}^{+}$signal.

Maxillaria notylioglossa $(2 \mathrm{n}=38)$ displayed three terminal and two subterminal $\mathrm{CMA}^{+} / \mathrm{DAPI}^{-}$bands and two proximal $\mathrm{CMA}^{-} / \mathrm{DAPI}^{+}$bands (Figure 1i and Figure 2), with up to 12 weaker $\mathrm{CMA}^{0} / \mathrm{DAPI}^{-}$and $\mathrm{CMA}^{+} / \mathrm{DAPI}^{0}$ bands sometimes being visible (not shown). Three of the four $5 \mathrm{~S}$ rDNA sites observed co-localized with the most evident $\mathrm{CMA}^{+} / \mathrm{DAPI}^{-}$bands (arrowheads in Figure 1i, j), showing that they are localized on two different chromosome pairs (Figure 2). Only a single pair of 45S rDNA sites (Figure 1j) was observed, terminally located and coincided with a weak $\mathrm{CMA}^{+}$band (arrows in Figure 1i).

Maxillaria desvauxiana $(2 \mathrm{n}=40)$ had two terminal $\mathrm{CMA}^{+} / \mathrm{DAPI}^{-}$bands (arrows in Figure 1k-1) and two terminal and one interstitial $\mathrm{CMA}^{+} / \mathrm{DAPI}^{0}$ bands (arrowheads in Figure 1k-1). Two terminal 45S rDNA sites and two terminal and one interstitial 5S rDNA sites were revealed by FISH (Figure $1 \mathrm{~m}$ ), all coinciding with $\mathrm{CMA}^{+}$bands.

\section{Discussion}

Only M. discolor had been previously investigated regarding chromosome number (Felix and Guerra, 2000), our results confirm this to be $2 \mathrm{n}=42$. The chromosome numbers reported here for the other three species $(2 n=38,40)$ were similar to the few other counts for the genus, i.e. three species with $2 \mathrm{n}=40$ and three with $2 \mathrm{n}=42$ (Felix and Guerra, 2000). Similarly, most better-studied orchid gen- era, such as the horticulturally important genera Laelia and Cattleya, show little variation in their chromosome numbers (see Felix and Guerra, 2000; 2005). However, our analysis with base-specific fluorochromes showed more pronounced karyological differentiation among the four Maxillaria species, including variation in the number and location of heterochromatic bands with both CMA and DAPI staining. Kao et al. (2001) observed that in Phalaenopsis species heterochromatin is a major source of variation in DNA content, while Kondo et al. (1994) and Hoshi et al. (1995) have shown that in the orchid genus $C y$ pripedium (which has large chromosomes) the amount and variability of heterochromatin was exceptionally high.

The bright $\mathrm{DAPI}^{+}$or $\mathrm{CMA}^{+}$bands observed in many plant genera (Guerra, 2000) reveal the presence of AT- or GC-rich repetitive DNA sequences, respectively (Schweizer, 1981). The unusual feature of the Maxillaria $\mathrm{CMA}^{+}$ bands was that all of them coincided with $5 \mathrm{~S}$ or $45 \mathrm{~S} \mathrm{rDNA}$. It is known that $45 \mathrm{~S}$ rDNA sites commonly stain positively after Giemsa C-banding or after direct staining with CMA, while $5 \mathrm{~S}$ rDNA sites are usually visualized only by in situ hybridization (Guerra and Felix, 2000; Vanzela et al., 2002; Carvalho and Guerra, 2002; Carvalho et al., 2005). This relationship between $5 \mathrm{~S}$ rDNA sites and $\mathrm{CMA}^{+}$bands was most evident in $M$. notylioglossa, in which the largest and brightest $\mathrm{CMA}^{+}$bands co-localized with $5 \mathrm{~S}$ rDNA sites instead of $45 \mathrm{~S}$ rDNA sites.

However, not all $5 \mathrm{~S}$ and $45 \mathrm{~S}$ rDNA sites in Maxillaria and in other genera generate $\mathrm{CMA}^{+}$bands, probably because the sites were too small to be detected with CMA (Zoldos et al., 1999; Marcon et al., 2003; 2005). Schmid and Guttenbach (1988) obtained positive mithramycin A staining of vertebrate nucleolus organizer region (NOR) in various species except humans, the authors attributing this exception to the low repeat number (40-46 repeats per locus) of human NORs. In Maxillaria, the rDNA site heteromorphism observed in three of the four species investigated may also be caused by differences in the number of rDNA repeats in some loci, as observed in Arabidopsis (Murata et al., 1997) and Trifolium (Ansari et al., 1999).

The co-localization of 5S rDNA sites with heterochromatic blocks has been rarely reported in plants, perhaps because only a few groups of plants have been simultaneously investigated by banding techniques and in situ hybridization with a 5S rDNA probe. In Hypochaeris (Cerbah et al., 1998; Ruas et al., 2005), Quercus (Zoldos et al., 1999) and Lilium species (Siljak-Yakovlev et al., 2003) both $5 \mathrm{~S}$ and $45 \mathrm{~S}$ rDNA sites were differentially stained by either CMA or C-banding followed by CMA/DAPI double-staining. In Clivia, $5 \mathrm{~S}$ and $45 \mathrm{~S}$ rDNA sites clearly coincided with Giemsa C-bands (Ran et al., 2001).

Loci of $5 \mathrm{~S} \mathrm{rDNA}$ are constituted of repeats containing a transcribed region of about $120 \mathrm{bp}$ and a non-transcribed spacer (NTS) of a very variable size, normally several hun- 
dred base pairs (Gottlob-McHugh et al., 1990). As the transcribed sequence is highly conserved, the difference in staining among $5 \mathrm{~S}$ rDNA sites in different genera might be due to NTS sequence divergence. It is interesting to note that in all plant species in which $5 \mathrm{~S}$ rDNA blocks have been observed the blocks within a chromosome complement seem to present the same reaction to the fluorochromes. This apparent intraspecific homogeneity may be due to the occurrence of interloci concerted evolution, as observed in cotton by Cronn (1996). However, differential intralocus homogenization has been demonstrated at the molecular level for several plant species, such as Arabidopsis (Cloix et al., 2000) and Nicotiana (Matyásek et al., 2002). Interestingly, the opposite situation is found in some vertebrates, in which different $5 \mathrm{~S}$ rDNA loci of a single karyotype may show very distinct families of NTS repeats and may be differentially stained by FISH using NTS specific probes (Martins and Galetti, 2001; Martins and Wasko, 2004).

The correlation between sites of repetitive DNA sequences and heterochromatic bands may also depend on factors other than DNA sequence. For example, in the fish species Astyanax scabripinnis terminal C-bands that hybridized with an AT-rich satellite DNA sequence stained positively with mithramycin A in one population and negatively in another population (Mantovani et al., 2004) and it has also been reported that in several Citrus species some $45 \mathrm{~S}$ rDNA sites are $\mathrm{CMA}^{+}$while others are $\mathrm{CMA}^{0}$ (Carvalho et al., 2005). Furthermore, the rDNA may apparently exist in distinct chromatin conformations determined by specific epigenetic codes, such as cytosine methylation and post-translational changes in histones (Neves et al., 2005), that could affect its reactivity with fluorochromes. These data suggest that, as with $5 \mathrm{~S}$ rDNA sites, other tandemly repetitive sequences may exist in chromosomes without forming heterochromatic blocks.

\section{Acknowledgments}

This study was supported by grants and fellowships from the Brazilian Agencies Conselho Nacional de Desenvolvimento Científico e Tecnológico (CNPq) and Fundação de Amparo a Ciência e Tecnologia do Estado de Pernambuco (FACEPE).

\section{References}

Ansari HA, Ellison NW, Reader SM, Badaevas ED, Friebe B, Miller TE and Williams WM (1999) Molecular cytogenetic organization of 5S and 18S-26S rDNA loci in white clover (Trifolium repens L.) and related species. Ann Bot 83:199206.

Brasileiro-Vidal AC, Cuadrado A, Brammer SP, Zanatta ACA, Prestes AM, Moraes-Fernandes MIB and Guerra M (2003) Chromosome characterization in Thinopyrum ponticum (Triticeae, Poaceae) using in situ hybridization with different DNA sequences. Genet Mol Biol 26:505-510.
Carvalho R and Guerra M (2002) Cytogenetics of Manihot esculenta Crantz (cassava) and eight related species. Hereditas 136:159-168.

Carvalho R, Soares Filho WS, Brasileiro-Vidal AC and Guerra M (2005) The relationships among lemons, limes and citron: A chromosomal comparison. Cytogenet Genome Res 109:276-282.

Cerbah M, Coulaud J and Siljak-Yakovlev S (1998) rDNA organization and evolutionary relationships in the genus Hypochaeris (Asteraceae). J Hered 89:312-318.

Cloix C, Tutois S, Mathieu O, Cuvillier C, Espagnol MC, Picard $\mathrm{G}$ and Tourmente $\mathrm{S}$ (2000) Analysis of 5S rDNA arrays in Arabidopsis thaliana: Physical mapping and chromosomespecific polymorphisms. Genome Res 10:679-690.

Cronn RC, Zhao X, Paterson AH and Wendel JF (1996) Polymorphism and concerted evolution in a tandemly repeat gene family: 5S ribosomal DNA in diploid and allopolyploid cottons. J Mol Evol 42:685-705.

Cuadrado A and Schwarzacher T (1998) The chromosomal organization of simple sequence repeats in wheat and rye genomes. Chromosoma 107:587-594.

Felix LP and Guerra M (2000) Cytogenetics and cytotaxonomy of some Brazilian species of Cymbidioid orchids. Genet Mol Biol 23:957-978.

Felix LP and Guerra M (2005) Basic chromosome numbers of terrestrial orchids. Plant Syst Evol 254:131-148.

Fuchs J, Strehl S, Brandes A, Scheizer D and Schubert I (1998) Molecular-cytogenetic characterization of the Vicia faba genome - Heterochromatin differentiation, replication and sequence localization. Chromosome Res 6:219-230.

Guerra M (2000) Patterns of heterochromatin distribution in plant chromosomes. Genet Mol Biol 23:1029-1041.

Guerra M and Felix LP (2000) O cariótipo de Nothoscordum pulchellum (Alliaceae), com ênfase na heterocromatina e nos sítios de DNAr. Bol Soc Argent Bot 35:283-289.

Guerra M, Kenton A and Bennett MD (1996) rDNA sites in mitotic and polytene chromosomes of Vigna unguiculata (L.) Walp. and Phaseolus coccineus L. revealed by in situ hybridization. Ann Bot 78:157-161.

Gottlob-McHugh SG, Lévesque M, MacKenzie K, Olson M, Yarosh O and Johnson DA (1990) Organization of the 5S rDNA genes in the soybean Glycine max (L.) Merrill and conservation of the $5 \mathrm{~S}$ rDNA repeat structure in higher plants. Genome 33:486-494.

Hoshi Y, Kondo K, Tanaka R and Karawasa K (1995) A comparison of C-banding pattern in nine species of Cypripedium (Orchidaceae). Kromosome 77:2636-2645.

Kao YY, Chang SB, Lin TY, Hsiehs CH, Chen YH, Chen WH and Chen CC (2001) Differential accumulation of heterochromatin as a cause for karyotype variation in Phalaenopsis orchids. Ann Bot 87:387-395.

Kondo K, Hoshi Y and Tanaka R (1994) Somatic chromosome differentiation in Cypripedium segawai Masamune and $C$. japonicum Thunberg. Cytologia 59:115-120.

Mantovani M, Abel LDS, Mestriner CA and Moreira-Filho O (2004) Evidence of the differentiated structural arrangement of constitutive heterochromatin between two populations of Astyanax scabripinnis (Pisces, Characidae). Genet Mol Biol 27:536-542. 
Marcon AB, Barros ICL and Guerra M (2003) A karyopyte comparison between two closely related species of Acrostichum. Am Fern J 93:116-125.

Marcon AB, Barros ICL and Guerra M (2005) Variation in chromosome numbers, CMA bands and 45S rDNA sites in species of Selaginella (Pteridophyta). Ann Bot 95:271-276.

Martins C and Galetti Jr PM (2001) Organization of 5S rDNA in species of the fish Leporinus: Two different genomic locations are characterized by distinct nontranscribed spacers. Genome 44:903-910.

Martins C and Wasko AP (2004) Organization and evolution of 5S ribosomal DNA in the fish genome. In: Williams CR (ed) Focus on Genome Research. New Science Publishers, Hauppauge, pp 289-318.

Matyásek R, Fulnecek J, Lim KY, Leitch AR and Kovarík A (2002) Evolution of 5S rDNA unit arrays in the plant genus Nicotiana (Solanaceae). Genome 45:556-562.

Murata M, Heslop-Harrison JS and Motoyoshi F (1997) Physical mapping of the 5S ribosomal RNA genes in Arabidopsis thaliana by multicolor fluorescence in situ hybridization in cosmid clones. Plant J 12:31-37.

Neves N, Delgado M, Silva M, Caperta A, Morais-Cecílio and Viegas W (2005) Ribosomal DNA heterochromatin in plants. Cytogenet Genome Res 109:104-111.

Pabst GFJ and Dungs F (1975) Orchidaceae Brasiliensis. $1^{\text {st }}$ volume. Brücke Verlag Kurt Schmersow, Hildenshein, 408 pp.

Pedrosa A, Sandal N, Stougaard J, Schweizer D and Bachmair A (2002) Chromosomal map of the model legume Lotus japonicus. Genetics 161:1661-1672.

Ran Y, Hammett KRW and Murray BG (2001) Phylogenetic analysis and karyotype evolution in the genus Clivia (Amaryllidaceae). Ann Bot 87:823-830.
Ruas CF, Vanzela ALL, Santos MO, Fregonezi JN, Ruas PL, Matzenbacher NI and Aguiar-Perecín MLR (2005) Chromosomal organization and phylogenetic relationships in Hypochaeris species (Asteraceae) from Brazil. Genet Mol Biol 28:129-139.

Schmid M and Guttenbach M (1988) Evolutionary diversity of reverse (R) fluorescent chromosome bands in vertebrates. Chromosoma 97:101-114.

Schweizer D (1981) Counterstain-enhanced chromosome banding. Hum Genet 57:1-14.

Shibata F, Matsusaki Y and Hizume M (2005) AT-rich sequences containing Arabidopsis-type telomere sequence and their chromosomal distribution in Pinus densiflora. Theor Appl Genet 110:1253-1258.

Siljak-Yakovlev S, Peccenini S, Muratovic E, Zoldos V, Robin O and Vallès J (2003) Chromosomal differentiation and genome size in three European mountain Lilium species. Plant Syst Evol 236:165-173.

Sumner AT (1990) Chromosome Banding. Unwin Hyman, London, $434 \mathrm{pp}$.

Vanzela ALL, Ruas CF, Oliveira MF and Ruas PM (2002) Characterization of diploid, tetraploid and hexaploid Helianthus species by chromosome banding and FISH with $45 \mathrm{~S}$ rDNA probe. Genetica 114:105-111.

Wanzenböck E-M, Schöfer C, Schweizer D and Bachmair A (1997) Ribosomal transcription units integrated via T-DNA transformation associate with the nucleolus and do not require upstream repeat sequences for activity in Arabidopsis thaliana. Plant J 11:1007-1016.

Zoldos V, Papes D, Cerbah M, Panaud O, Besenborfer V and Siljak-Yakovlev (1999) Molecular-cytogenetic studies of ribosomal genes and heterochromatin reveal conserved genome organization among 11 Quercus species. Theor Appl Genet 99:969-977.

Associate Editor: Yatiyo Yonenaga-Yassuda 